\title{
La palabra complementada (cued speech). De la percepción visual del habla a la comprensión y producción de la palabra
}

\section{Santiago Torres}

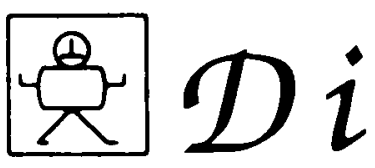

Los sistemas paraverbales o que acompañan al habla son necesarios a todo hablante "normal». Algunos pueblos, como los latinos (frente a los anglosajones, por ejemplo), bacemos uso generoso y eficiente de ellos. Un trabajo sistemático con ellos ba permitido poner en marcha un método eficaz para la educación del lenguaje oral en niños sordos. En este articulo se expone en detalle el sistema, sus implicaciones y eficacia y los pasos a dar para su aplicación.

30 segundos me son suficientes para que un sordo profundo comprenda para qué sirve la PC.

Pero no me bastan 30 minutos para bacérsela comprender a un oyente.

(Cornett, 1988)

\section{PRETEST}

Intente descifrar estas dos palabras en los labios (sin otra ayuda que la visión. Se articulan en silencio):

1) Mamá.

2) Papá.

Ahora, aprenda estas claves:

La mano abierta de forma natural y puesta al lado de la cara designa la sílaba $\mathrm{Ma}$.

La mano cerrada con el índice extendido y puesta igualmente al lado de la cara, designa la sílaba $\mathrm{Pa}$.

Se repiten las dos palabras con lectura labiofacial (LLF) + palabra complementada ( $\mathrm{PC}$ ) (se articulan sin voz, como en la primera presentación).

Si Ud. ha captado la relación existente entre complementos manuales y discriminación de fonemas confundibles en los labios, usted ha comprendido para qué sirve la PC. 


\section{JUSTIFICACION DE LA PC}

La Palabra Complementada (en adelante PC) está justificada ante el fracaso del oralismo y la comprobación científica de la necesidad y al mismo tiempo insuficiencia de la lectura labiofacial (en adelante LLF) (Perelló, 1978). La PC en sincronía con la LLF eleva la discriminación visual de la palabra a niveles próximos al $100 \%$ (Nicholls, 1979). Convencidos de la necesidad de la LLF, tanto en sordos como en oyentes para adquisición de ciertas tareas metalingüísticas (Alegría \& al., 1985, 1986, 1987; Liberman, 1985; Morais, Alegría, Content, 1987; Asensio, 1988; Sánchez Miguel, 1990) la PC nunca la sustituye, sino que en todo momento la refuerza, como tendremos ocasión de ver a lo largo de este trabajo. Es más, la LLF es parte integrante y principal de la PC. En suma, la PC es LLF más complementos manuales. Esto hace que la PC sea filosófica y funcionalmente un sistema oral (Cornett, 1991).

\section{PRESUPUESTO BASICO}

Si todos los fonemas del habla se vieran con claridad en los labios del hablante, el niño sordo aprendería a hablar sin dificultad y al ritmo del oyente. Aprendería a hablar de forma natural, solo que usando la vista en lugar del oído (Cornett, 1967).

$\mathrm{Al}$ acto de ver la palabra en la boca del hablante se llama, comúnmente, LLF. La LLF, en la que se basan la mayoría de los métodos oralistas y de la que no prescinde ningún sordo, es tan imprescindible como insuficiente, alcanza sólo el $33 \%$ de discriminación fonémica y requiere conocimientos previos del idioma (Bravo Castell, S. \& al., 1987; Perelló, 1978). Estas dos condiciones hacen que no sea un instrumento idóneo para el desarrollo natural del aprendizaje verbal. Por esta razón, desde Ponce de León hasta nuestros días se ha venido intentando un sistema que hiciera visible el lenguaje al sordo. La PC lo ha logrado.

\section{DEFINICION DE LA PALABRA COMPLEMENTADA}

La Palabra Complementada es un sistema que, mediante ocho configuraciones de la mano realizadas en tres posiciones (lado, barbilla, garganta) respecto al rostro, más la información aportada por la lectura labiofacial, clarifica visualmente el lenguaje hablado. Cuando los sonidos o sílabas del habla son confundibles en los labios, los complementos manuales son distintos para clarificarlos. $\mathrm{Y}$ al revés, los complementos son iguales cuando los sonidos o sílabas se ven distintos en los labios.

La PC es la rẹspuesta, hoy por hoy más rentable y económica, al mayor problema con que topan los sordos: la percepción de la palabra como requisito previo para la comprensión y producción verbal.

Se sabe que a partir de un cierto grado de sordera, en concreto a partir de los $90 \mathrm{~dB}$ de pérdida, el equipamiento protésico no sirve para que las informaciones auditivas, que recibe el sordo, tengan calidad suficiente para percibir de modo satisfactorio el lenguaje hablado $y$, como consecuencia, 
la comprensión del mensaje se hace difícil y a veces imposible. El recurso a la LLF es, por tanto, indispensable, pero insuficiente.

La lectura labiofacial proporciona al sordo profundo informaciones incompletas o ambiguas, pues muchos fonemas distintos se articulan con idénticos movimientos labiales y otros fonemas ni siquiera pueden verse. Asi, pues, ciertos sonidos, v.gr.: $/ p-b-m / o / t-d-n /$, tienen formas casi idénticas en los labios, o al menos tan similares que la lectura labial se hace muy difícil.

Esto quiere decir que si al sordo profundo se le dice papá, mamá o $b a b a$, fuera de contexto, tendrá que adivinar qué consonante ha sido dicha con riesgo de acertar una de cada tres veces, o sea, el porcentaje atribuido al azar.

Por esta razón, la LLF es un ejercicio muy fatigoso y ambiguo. Es por esto que no se puede confiar en la LLF para la percepción de los estímulos lingüísticos y el posterior desarrollo de la comprensión y producción verbal, dadas las pocas y deficientes informaciones que proporciona.

La PC o Cued Speech ha venido a solucionar este serio problema. Una vez solucionado el problema de la ambigüedad orofacial a edades tempranas, el niño sordo lo tiene todo mucho más fácil para adquirir el lenguaje $y$ el habla de forma casi natural.

\section{HISTORIA DE LÁ PC}

El Dr. R. Orin Cornet llegó a la Universidad Gallaudet de Washington con la presunción de que los sordos leían mucho (Cornett, 1967). Pronto se dio cuenta del poco interés que los sordos tenían por la lectura, al convertírseles en una pesada carga por su insuficiente competencia lingüística primaria. Lo lógico sería, pensaba Cornett, que la lectura fuera para los sordos un medio privilegiado de información. Constatar que los sordos leían poco y mal fue su primera y gran decepción. Pensó en las causas y descubrió que el sordo profundo no es lingüísticamente competente por falta de vocabulario y escasa asimilación de la morfosintaxis (Conrad, 1977, 1979). Y todo ello por la escasa información confiable recibida en la primera infancia. La PC es ideada para que el niño sordo, desde su más temprana edad, perciba de forma inconfundible los estímulos verbales en un ambiente de naturalidad.

Para lograr que los sordos aprendan a hablar de la forma más parecida posible al aprendizaje de los oyentes, le pareció que era necesario proponer una forma eficaz que permitiera percibir todo el discurso hablado a través de la vista, igual que los oyentes perciben todo lo bablado a través del oído. $\mathrm{Al}$ comprobar que la lectura labial representa de forma muy incompleta el discurso hablado, trabajó para poner a punto un sistema complementario de apoyo a la lectura labial, que aportara las informaciones que faltan a la LLF eliminando al mismo tiempo todas sus ambigüedades.

Cornett actuó con una genialidad nada común. Intuyó que la vista podía suplir al oído y creó un sistema eficaz para que los sordos vean la palabra. Este fue el comienzo de una serie de investigaciones que han demostrado que también la vista, en el caso de los oyentes, está implicada en la adquisición de tareas metalingüísticas, sin cuyo dominio es muy difícil lle- 
gar al dominio de la lectoescritura (Alegria \& al., 1985, 1986, 1987; Liberman, 1985; Morais, 1987; Asensio, 1988; Sánchez Miguel, 1990).

En la PC, estas informaciones complementarias son proporcionadas por la mano, que realiza, en sincronía con los labios, claves (cues, en inglés) o configuraciones manuales - también llamados kinemas-. Cada kinema comprende dos parámetros: la posición de la mano respecto al rostro y la forma de los dedos. La combinación figura + posición (forma y lugar), corresponde a una sílaba en sentido amplio, pues mejor sería hablar de secuencia C $+\mathrm{V}$. Para simplificar diremos que la PC es un sistema silábico.

La regla de oro de la PC es que todos los fonemas con igual visema (semejantes y confundibles en los labios) van acompañados de un kinema diferente, mientras que los fonemas diferenciables en los labios son complementados con el mismo kinema. De esta manera, a cada una de las tres posiciones (lado, barbilla, garganta) corresponden vocales fácilmente diferenciables en los labios, y a cada una de las 8 configuraciones o figuras de los dedos corresponde un grupo de consonantes bien diferenciables en los labios, v.gr.: la figura 5 , a la que corresponden los fonemas $/ \mathrm{m}, \mathrm{f}, \mathrm{t} /$. Dicho brevemente, a igual visema corresponden distintos kinemas, a igual kinema corresponden distintos visemas.

Ni la imagen labial sola, ni el complemento manual solo permiten la identificación inequivoca de un fonema. Por el contrario, el fonema queda enteramente definido y puede ser reconocido sin ambigüedad mediante la asociación de la imagen labial y el complemento manual. La PC es, pues, inseparable de la lectura labial; esto marca la diferencia específica entre la PC y otras ayudas manuales.

\section{ELEMENTOS FORMALES DE LA PC (cfr. Tabla 1)}

La PC, en la versión española, comprende 3 posiciones de la mano respecto al rostro - lado, barbilla, garganta-, y 8 figuras o configuraciones de los dedos (Torres, 1988), más las aportaciones de la LLF.

Las 3 posiciones de la mano, respecto al rostro, sirven para discriminar las vocales.

posición lado: vocal /a/

posición barbilla: vocales $/ e, \mathrm{o} /$

posición garganta: vocales $/ \mathrm{i}, \mathrm{u} /$

Las 8 figuras de la mano, sirven para discriminar las consonantes:

Fig. $1 / \mathrm{p} / / \mathrm{d} / / \check{\mathrm{z}} /$

Fig. $2 / \mathrm{k} / / \mathrm{n} / / \mathrm{d} / / \mathrm{v} /$

Fig. $3 / \mathrm{x} / / \mathrm{r} / / \mathrm{s} /$

Fig. $4 / \mathrm{b}, \mathrm{v} / / \mathrm{n} / / \mathrm{xw} /$

Fig. $5 / \mathrm{m} / / \mathrm{f} / / \mathrm{t} / /-1$

Fig. $6 / 1 / / \mathrm{w} /$

Fig. $7 / \theta / / g / / \bar{r} / / l, y /$

Fig. $8 / \hat{c} / / \mathrm{j} / / \mathrm{p} /$

RECUERDE

Las POSICIONES se relacionan siempre con las VOCALES y SEMIVOCALES

Las FIGURAS se relacionan siempre con las CONSONANTES Y SEMICONSONANTES 


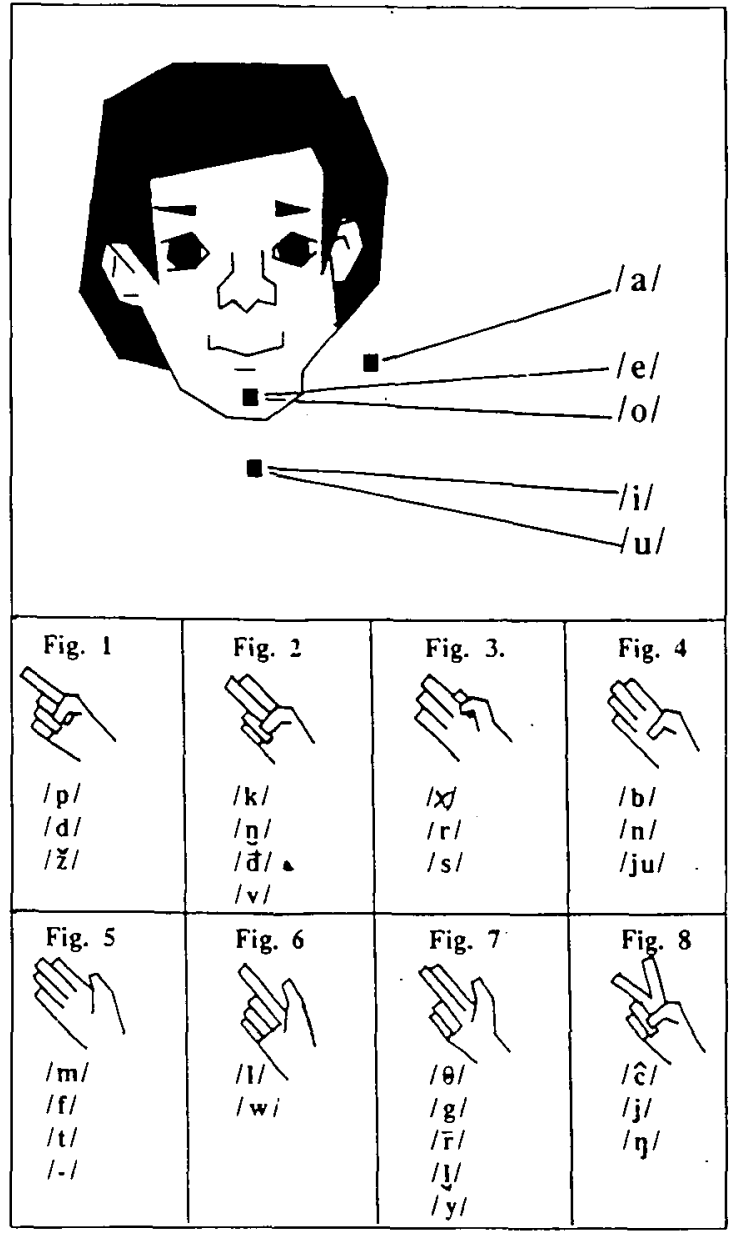

VERSION ESPAÑOLA DEL CUED SPEECH LA PALABRA COMPLEMENTADA

Además de las 3 posiciones y las 8 figuras hay 3 movimientos de la mano, que sirven para discriminar la secuencia $\mathrm{c}+\mathrm{v}$ (mov. adelante) versus la aparición de una consonante aislada (mov. flick) y la prosodia, duración de cada fonema concreto y ritmo del habla (mov. relax).

Movim. adelante: acompaña a toda sílaba directa.

Movim. flick: acompaña a toda consonante aislada. bla.

Movim. relax: marca la prosodia y contribuye a la comprensión del ha-

Los 3 movimientos tienen que contribuir a matizar con precisión la duración, il tempo, correspondiente a cada fonema y a contraponer la sílaba frente a la consonante aislada.

El Dr. Cornett terminó rechazando una orientación que le pareció lógica al principio, esto es, la perspectiva fonética, que consistía en representar los fonemas de forma que indicaran las propiedades fonéticas de los sonidos emitidos, v.gr.: sordas, sonoras, fricativas, etc. En efecto, las pruebas 


\section{RECUERDE}

El movimiento ADELANTE acompaña siempre a la SILABA DIREC$T A$, o mejor, a la secuencia $C+V$, ej.: la-ma-má-se-va. Marca el tiempo de emisión.

El movimiento FLICK acompaña siempre a la consonante de las SILABAS INVERSAS y siempre que una consonante quede aislada y sin vocal, ej.: e-S, do-S, má-S, etc. La consonante aislada es breve en el tiempo, lo que queda resaltado con el movimiento flick.

efectuadas demostraron que no era posible conjugar a la vez un sistema muy preciso en recepción y al mismo tiempo fonético. Por esta razón el agrupamiento de los fonemas en las distintas figuras no corresponde a las propiedades fonéticas o acústicas del habla (Cornett, 1976).

En segundo lugar, el Dr. Cornett buscó que el contraste visual entre los diferentes fonemas, agrupados en un kinema, fuera el máximo, conjugando este principio con otro no menos importante, el de la economía de energia, que consiste en tener en cuenta la frecuencia de los fonemas asignados a cada configuración (Cornett, 1967). Por ejemplo, la configuración 5 (todos los dedos de la mano extendidos, mano abierta) se emplea con el grupo de fonemas más frecuente, ya que es la forma más fácil de hacer, puesto que no exige doblar ningún dedo. Las demás formas han sido asignadas de manera que el grupo de menor frecuencia corresponda a la forma más difícil de hacer. Esto contribuye a mitigar la fatiga del locutor, que complementa al tiempo que habla.

Otros dos elementos fueron tenidos en cuenta. Por una parte, el sistema está hecho de tal manera que proporciona una percepción extremadamente precisa de los fonemas. La precisión de la percepción que se logra es equivalente a la obtenida por los oyentes mediante la sola audición. Con la PC pueden representarse las variaciones de los distintos locutores de una misma lengua, v.gr.: diferencia en la pronunciación de $/ /$ e $/ y /, o / d /$ y $/ \partial /$ por ejemplo. Por otra parte, el sistema es compatible en las distintas lenguas, aunque para hacerlo compatible hubo que sacrificar otros criterios de concepción. De hecho, actualmente, la PC está adaptada a unas 52 lenguas.

\section{BASES TEORICAS DE LA PC}

- Es un sistema oralista,

- es un sistema basado en la segmentación silábica,

- es un sistema basado en la fonética del habla,

- es un sistema práctico y fácil de aprender,

- es la oferta más económica de un lenguaje razonado,

- es la posibilidad de presentar el habla de forma natural, progresiva y funcional,

- es una vía sustitutoria del oído,

- es un sistema compatible con cualquier programa logopédico,

- es un sistema complementario de cualquier método en uso.

La unidad básica de un sistema de representación visual del discurso hablado debe ser la silaba. De otra manera, sería imposible sincronizar esta 
TABLA I

Clasificación de los fonemas del español en orden decreciente de frecuencia (Quilis y Esgueva, 1980)

\begin{tabular}{|c|c|c|c|}
\hline Vocales & $\%$ & Consonantes & $\%$ \\
\hline $\begin{array}{l}\mathrm{e} \\
\mathrm{a} \\
\mathrm{o} \\
\mathrm{i} \\
\mathrm{u}\end{array}$ & $\begin{array}{r}14,67 \\
12,19 \\
9,99 \\
7,38 \\
3,33\end{array}$ & 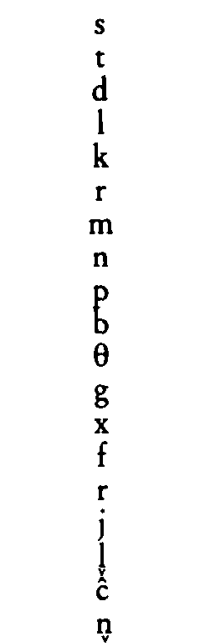 & $\begin{array}{l}8,33 \\
4,54 \\
4,24 \\
4,24 \\
3,99 \\
3,26 \\
3,06 \\
2,78 \\
2,77 \\
2,38 \\
1,46 \\
0,95 \\
0,57 \\
0,56 \\
0,44 \\
0,41 \\
0,38 \\
0,37 \\
0,25\end{array}$ \\
\hline
\end{tabular}

Con más del $7 \%$ de frecuencia están, por orden decreciente, los fonemas $/ \mathrm{e} / \mathrm{a} / \mathrm{o} / \mathrm{s} /$ $/ \mathrm{i} /$, que representan el $52,54 \%$ del total. Con menos del $5 \%$ están, por orden decreciente, $/ \mathrm{t} / / \mathrm{d} / / \mathrm{l} / \mathrm{k} / / \mathrm{u} / / \mathrm{r} / / \mathrm{m} /$, que representan el $31,49 \%$. Con menos del $2 \%$ están $/ \mathrm{p} /, / \mathrm{p} /$, $/ \mathrm{b} /, / \theta /$, que agrupan el $11,3 \%$ del total. El resto no llegan a sumar ni el $5 \%$ restante. Existe claro predominio de las vocales $/ \mathrm{e} / \mathrm{/a} / \mathrm{o} /$ sobre el resto de los fonemas, alcanzado el $77,47 \%$ en relación al conjunto.

representación visual con la percepción de los segmentos del habla. Se ha demostrado que la PC permite complementar la sílaba gracias a la combinación posición de la mano-configuración de los dedos. En este aspecto se diferencia de la dactilología, que equivale a un deletreo lento, en comparación con el ritmo del habla y que no representa las características fonológicas y metalingüísticas del lenguaje.

Al basarse en la sílaba como unidad básica de representación visual, garantiza:

a) la posibilidad de acomodarse en velocidad al discurso normal,

b) el entrenamiento en segmentación silábica, anterior y básico a la segmentación fonológica,

c) la posibilidad del aprendizaje verbal natural al hacer visible todo el discurso.

Al no tener los kinemas significado alguno por sí solos, se diferencia de los sistemas gestuales con las ventajas que esto conlleva para la lectoescritura.

Al no sustituir, sino necesitar de la LLF, ésta queda clarificada en cualquier acto de habla y reforzada con el uso de LLF + PC. La información aportada por los labios debe ser parte integrante y principal del sistema, y esto por dos razones: 
a) La mano es demasiado torpe como para permitir comunicar un mensaje completo a la velocidad del habla coloquial. Los conocimientos de física que tiene el Dr. Cornett le han permitido calcular que la mano puede llegar a producir la mitad de la información del lenguaje hablado y los labios la otra mitad.

b) El sistema debe obligar al niño a leer en los labios y de esta manera contribuir a que sea un buen lector labiofacial con el fin de no crearle una dependencia total en relación con los complementos.

Con la PC se ha buscado y se ha logrado un sistema simple en el aprendizaje y rápido en el afianzamiento. Era preciso diseñar un sistema que en pocas semanas los padres pudieran aprenderlo y comunicarse con su hijo; el sistema debía poder ser captado, en lo que a recepción se refiere, por un niño sordo menor de dos años de edad.

Al concebirse para ser usado en familia, se ha conseguido que esté libre de cultura, es independiente del nivel cultural de quien lo practica.

Ha sido ideado para que contribuya al desarrollo natural del lenguaje, por eso el sistema es tan simple que puede ser captado por un niño menor de 2 años de edad.

Destinado a ser visto, se logró que los kinemas fueran distinguibles a 10 metros de distancia.

Si el niño oyente aprende el lenguaje oyendo hablar, se buscaba un sistema que presentara a la vista la misma cantidad y grado de discriminación, al menos, de estímulos verbales percibidos por el oído sano. Con la PC se puede llegar al $100 \%$ de discriminación visual del habla (Clarke y Ling, 1976; Ling y Clarke, 1975). Un oyente sano también puede llegar al $100 \%$ de discriminación, pero no es frecuente alcanzar esta tasa mediante la audición. Con la PC el niño sordo aprenderá a hablar desde lo que se oye, desde lo que se ve, igual que ocurre con el niño oyente.

Finalmente, resaltar que la PC no es un sistema o método mímico; no es un método ortofónico, aunque contribuye a la larga a mejorar la ortofonía; no es un sistema gestual, no desplaza, $n i$ interfiere, $n i$ sacrifica otros métodos o sistemas; no compromete la filosofía oralista (Cornett, 1986).

\section{DESTINATARIOS DE LA PC}

La PC se dirige, en primer lugar, a los padres oyentes con el fin de hacer inteligible su lenguaje al sordo (Pirnay-Schira \& al., 1987). El grado de sordera no constituye un criterio decisivo para la aplicación de la PC. Es verdad que la PC beneficiará más a un sordo profundo que a otro que no lo sea tanto. Pero en todos los casos en los que el recurso a la LLF, parcial o total, sea necesario, la ayuda aportada al sordo por la PC se revela positiva desde el momento en que todas las ambigüedades quedan suprimidas, contribuyendo a centrar más la atención y a disminuir la fatiga.

La PC nació con vocación de reducir el problema de la comunicación temprana entre los niños sordos profundos y sus padres oyentes - el $90 \%$ de los niños sordos profundos son hijos de padres oyentes- (Valmaseda, 1987). A este respecto, Cornett es tajante: Si es necesario elegir entre el uso de los padres o el uso de los profesores, el uso que hacen los padres es más importante. Pero, si los padres no complementan, el uso de la PC por los 
profesores siempre aportará algo al niño, aun con el riesgo de que los resultados no se vean a corto plazo (Cornett, 1986).

Lo ideal es, evidentemente, que empleen la PC todas las personas que se relacionan con el niño. Esto ha quedado claro en las investigaciones desarrolladas por el Equipo del Centro Comprendre et Parler de Bruselas, en colaboración con el Laboratorio de Psicología Experimental de la Universidad Libre de Bruselas (Pèrier \& al., 1984, 1985, 1986, 1987). Un aspecto de estas investigaciones ha consistido en cuantificar objetivamente la mejora perceptiva alcanzada por niños sordos educados en LLF y PC frente a los niños educados sólo mediante LLF.

Para ello, 55 niños han sido sometidos a una prueba llamada prueba de recepción verbal, que consistía en presentarles una frase, bien mediante sólo LLF, o bien con LLF y PC. Al niño se le presenta una lámina con 4 imágenes. El niño debe indicar la imagen correspondiente a la frase que ha entendido. Los items han sido presentados en grupos de 4 imágenes que representan tres tipos de frases ${ }^{1}$ :

- de fácil diferenciación entre sí,

- de dificultad media para ser diferenciadas,

- difíciles de diferenciar entre sí.

Los 55 niños de la muestra fueron separados en 3 grupos diferentes según que tuvieran PC:

- sólo en casa (grupo C: 7 sujetos),

- sólo en la escuela (grupo E: 41 sujetos),

- tanto en casa como en la escuela (grupo $C+E: 7$ sujetos).

¿Qué porcentaje de mejora se ha constatado comparando los resultados alcanzados en PC frente a los obtenidos mediante sólo LLF?

Los 55 sujetos de la muestra, globalmente considerados, mejoran en $12,90 \%$.

El grupo $\mathrm{E}$ obtiene una ganancia global de $9,87 \%$.

El grupo $C$ obtiene una ganancia global de $17,60 \%$.

$\mathrm{El}$ grupo $\mathrm{C}+\mathrm{E}$ obtiene una ganancia global de $26 \%$.

Los autores de esta investigación puntualizan que el material de la prueba ha sido concebido de manera que no introduzca ninguna dificultad lingüística, por una parte, y por otra, de manera que el contexto no influya nada. En la vida real, el contexto se da y ayuda.

Si nos fijamos en la condición difícil, se ve que los porcentajes son todavía más interesantes, ya que en esta situación difícil, los porcentajes de partida son bajos, mientras que en las condiciones fáciles, los resultados son ya importantes de salida.

En la situación difícil, se observa:

- Para el grupo E, una mejora de $16,08 \%$.

- Para el grupo C, una mejora de $22 \%$ (se pasa del $44 \%$ al $66 \%$ de reconocimiento). $69 \%$ ).

- Para el grupo C + E, una mejora del $42 \%$ (se pasa del $27 \%$ al

Estos resultados confirman que el uso de la PC en casa y en la escuela es la situación ideal. 


\section{PC Y COMUNICACION}

\subsection{Recepción y producción de la palabra}

Hay que tener en cuenta que la PC es sólo una técnica de ayuda a la recepción de la lengua hablada.

No se trata de una ayuda para articular mejor o interiorizar los secretos del acento y la prosodia, como pueda ser en estos aspectos el Método Verbotonal. Es importante precisar este punto. Por una parte, para evitar las confusiones y no exigir a la PC aquello para lo que no ha sido ideada; por otra parte, para poder medir qué nivel de complementariedad aporta la PC a otros métodos (Abroc, 1980; Pèrier, 1984).

Dicho esto, está claro que la PC puede ser utilizada subsidiariamente con provecho por el oyente para rectificar el error articulatorio que comete el niño sordo al hablar, o para saber con precisión en entrenamiento auditivo, por ejemplo, lo que el niño sordo ha querido decir, o para que el propio niño sordo no haga reducciones silábicas o segmentarias en sus producciones orales.

\subsection{Recepción y comprensión de la palabra}

Se ha insistido en que la PC no es más que una ayuda al servicio de la recepción del lenguaje hablado. Pero recepción no equivale a comprensión. No hay que olvidar que los códigos de la PC son totalmente abstractos. En sí mismos no significan nada. La información aportada por los kinemas unida a la aportada por la LLF constituyen una información completa y sin ambigüedades en el plano de la forma, pero no son portadoras de significado más que cuando los vocablos complementados son conocidos de antemano. Ahora bien, el uso de la PC desde temprana edad contribuye al desarrollo del lenguaje (Nicholls, 1979; Nicholls y Ling, 1982; Destombes, 1981).

\subsection{PC y adquisición de la morfosintaxis}

La PC se revela particularmente útil en lo que concierne a la adquisición de las estructuras morfosintácticas de la lengua por parte del niño sordo, que sigue un proceso de adquisición casi idéntico al del niño oyente.

En efecto, el complementar toda la cadena hablada da al niño sordo una representación visual de las características fonológicas, gramaticales y sintácticas de la lengua. Gracias a la PC, el niño ve los marcadores sintácticos, las desinencias verbales, etc. Esto le permite hacer hipótesis sobre el funcionamiento de la lengua, como lo demuestran las buenas faltas cometidas a menudo por un niño expuesto a la PC, que dirá, como un niño oyente, yo ero bueno, yo sabo la lección, etc. A partir de aquí, el trabajo de construcción de la lengua, largo, costoso y artificial, que deben seguir los profesionales con un niño no sometido a la PC, se facilita considerablemente.

\section{CONCLUSION}

La PC, hay que insistir en ello, es una ayuda específica. No es una solución milagrosa, ni lo único de que disponemos para la educación del sordo. 
En aspectos que no son competencia de la PC, especialmente los de la producción de la palabra y el aprovechamiento de los restos auditivos, pueden y deben ser usados otros métodos y técnicas, además de la PC (Pèrier, 1986).

La PC es una ayuda cuya utilización debe formar parte de una estrategia global de comunicación con el niño sordo.

Así, no sólo es necesario vigilar que la recepción del habla esté acompañada de la comprensión, sino también que la producción verbal del niño sordo sea suficientemente rica en cantidad y calidad. Está claro que el vehículo de transmisión no puede sustituir la materia a transmitir: si el mensaje es pobre, seguirá siendo pobre con cualquier sistema o método, incluida la PC. Pero si el modo de empleo es racional y pragmático, si la ayuda está puesta al servicio de una voluntad real de comunicación con el niño sordo, entonces el uso de la PC produce efectos que van más allá de su carácter estrictamente instrumental. Al disponer de una herramienta eficaz, los padres se sienten arropados en su papel de padres, refuerzan su confianza en sus competencias y las de su hijo. El aumento del nivel de ambición y de exigencias que esto conlleva, entraña un enriquecimiento progresivo de los intercambios verbales. Desde este punto de vista, más que una herramienta, la PC se convierte en un motor de progreso en el camino del niño sordo hacia la adquisición del lenguaje.

\section{Referencias}

Abroc, J. L. (1980). «Quelques aspects de l'apport du Cued Speech dans la réhabilitation de l'enfant handicapé auditif». Bull. Audiophonol., 11/3, 41-78.

Alegria, J. (1985). «Por un enfoque psicolingǘstico del aprendizaje de la lectura y sus dificultades". Infancia y Aprendizaje, 29, 79-99.

Alegria, J. y LEYBAERT, J. (1986). Rôle du LPC dans l'identification de mots chez l'enfant sourd: théorie et données préliminaires. Actes du Colloque Systèmes de communication alternatifs et augmentés. Liège.

ASENSIO, M. (1988). Los procesos de lectura en los deficientes auditivos. Tesis doctoral. UAM.

Clarke, B. R. y LiNG, D. (1976). "The effects of using cued speech: a follow-up study». The Volta Rev., 78, 23-35.

CORNeTt R. O. (1967). "Cued Speech". Am. Ann. Deaf, 112, 3-13.

-- (1980). "Compte rendu des journées d'études du LCC". En: Liaison LCC, n. 4. París.

- (1982). "Le Cued Speech». En: Destombes F. (ed.). Aides manuelles à la lecture labiale et perspectives d'aides automatiques. Centre scientifique IBM-France, París.

- (1986). La PC visualmente para los sordos. En Torres, S. Logopedia y Nuevas Tecnologías. Madrid. OEI-Apanda.

- (1991). Clarifying Statements. (Comunicación personal, no publicada).

DESTOMBES, F. et al. (1981). Le Cued Speech et la réception du langage parlé. Traducción de Cued Speech and the reception of spoken language de G. NICHOLLS. París, ALPC.

LiBERMAN, I. y SHANKWEILER, D. (1985). "Phonology and the problems of learning to read and write. RASE, 6, 8-17.

Ling, D. y Clarke, B. (1975). "Cued Speech: An evaluative study». Am. Ann. Deaf, 120, 480-488.

MarCHeSI, A. (1987). Desarrollo cognitivo y lingüístico de los niños sordos. Madrid. Alianza Editorial.

MORAIS, J.; AlEGRIA, J. y CONTENT, A. (1987). "The relationship between segmental analysis and alphabetic literacy: an interactive view». European Journal of Cognitive Psycho$\log y, 7,5.1-24$.

NiCHOLLS, G. H. (1979). Cued Speech and the reception of spoken language. Master's Thesis, McGill University, Montreal.

NiCHOLLS, G. H. y LING, D. (1982). «Cued Speech and the reception of spoken language». J. Speech and Hear. Res., 25, 262-269.

Perello, J. y Tortosa, F. (1978). Sordomudez. Barcelona, Científico-Médica.

PERIER, O. (1984). «Position du Cued Speech par rapport aux autres méthodes». Revue générale de l'Enseignement des déficients auditifs, 76, 99-113. 
Perier, O.; Charlier, B.; Hage, C. y Alegria, J. (1985). «Evaluation of the effects of prolonged Cued Speech practice upon the reception of spoken languagem. Annales du Congrés Mondial de l'Education des Sourds. Manchester.

Perier, O.; Bochner-Wuidar, A.; Capouillez, J. M.; Everarts, B.; Hage, C. y Michiels, J. (1986). The combination of Cued Speech and Signed French to improve spoken language acquisition by young deaf children. En: Tervoort B. (ed.). Signs of Life. Proceedings of the Second European Congress on Sign Language Research. Amsterdam, 194-199.

Perier, O. y Temmerman P. (1987). L'enfant à auditioné deficiente: aspects médicaux, éducatifs, sociologiques et psychologiques. Bruxelles, Acta Oto-Rhino-Laryngologica Bélgica, vol. 41,n. ${ }^{\circ} 2,307-314$ y 332-341.

Pirnay-Schira, A. M., Dissoubray, R.; Bazureau, B.; SpinetTa, N. y J. C. (1987). Guide des parents pour l'éducation de l'enfant sourd à l'aide du Language Parlé complété. Adaptación francesa del Handbook por parents de M. E. Wenegar y O. Cornett. Les Cabiers $d u$ LPC, 1. París.

SÁNCHEZ Miguel, E. (1990). Por qué los niños no leen bien. En A. Marchesi, C. Coll y J. Palacios. Necesidades educativas y aprendizaje escolar. Madrid, Alianza.

TORres Monreal, S. (1988). La Palabra Complementada. Madrid, CEPE.

VALMASEDA, M. (1987). Interacción, desarrollo social y características de personalidad del niño sordo. En Marchesi, A. El desarrollo cognitivo y lingǘítico de los niños sordos. Madrid. Alianza Psicología.

VINTER, S. (1981). «Le Cued Speech». Bull. Audiophonol. 13, supp. 3, 1-37.

\section{Notas}

frase):

${ }^{1}$ Ejemplos de frases de mediana dificultad (ambigüedad sólo en la segunda mitad de la

- la valise est ouverte /la valise est toute verte

- la botte est ouverte / la botte est toute verte

Ejemplos de frases difíciles (posible confusión labial en todos los términos de la frase):

- il monte le gâteau./ il montre le gâteau

- il montre le cadeau / il monte le cadeau 
La palabra complementada (Cued Speech). De la percepción visual del habla a la comprensión y producción de la palabra. $S$. Torres. CLE, 1991, 10, 71-83.

\section{Resumen}

La Palabra complementada es un sistema que, mediante ocbo configuraciones de la mano realizadas en tres posiciones (lado, barbilla, garganta) respecto al rostro, más la información aportada por la lectura labiofacial, clarifica visualmente el lenguaje bablado. Esta conjunción de complementos manuales más lectura labiofacial hace que el sistema sea filosófica y funcionalmente oral. Está indicado su empleo en estimulación del lenguaje oral con sordos en general y con sordos profundos en particular. En este artículo se presentan las caracteristicas que conforman la Palabra Complementada y se argumenta cientificamente las ventajas de su empleo. Este sistema está disponible actualmente en 52 lenguas, es conocido en todo el mundo y gana adeptos día a dia por su funcionalidad, así como por su compatibilidad y complementariedad con cualquier otro método o sistema en uso.

Datos sobre el autor: Santiago Torres Monreal es profesor de psicología en el Dpto. de Psicologia Básica de la Universidad de Málaga. La última década ha estado consagrado al proceso de desarrollo verbal del niño sordo. Desde 1984 se ha convertido en representante reconocido en España de la Palabra Complementada.

Dirección: Universidad de Málaga. Dpto. Ps. Básica. Campus de El Egido. 29013 Málaga. Tfno.: 952/243969.

(C) De todos los artículos. Deberá solicitarse por escrito autorización de CLE para el uso en forma de facsímil, fotocopia o cualquier otro medio de reproducción. CLE se reserva el derecho de interponer las acciones legales necesarias en aquellos casos en que se contravenga la ley de derechos de autor. 